\title{
Desenvolvimento de Estômago de Bezerros Holandeses Desaleitados Precocemente ${ }^{1}$ \\ Paulo Afonso Carvalho², Luis Maria Bonnecarrère Sanchez ${ }^{3}$, Julio Viégas ${ }^{3}$, João Pedro Velho ${ }^{4}$, Gilberto Cardoso Jauris ${ }^{5}$, Marcos Bittencourt Rodrigues ${ }^{6}$
}

\begin{abstract}
RESUMO - O presente estudo foi desenvolvido com o objetivo de avaliar, em diferentes idades da fase inicial da vida produtiva, o desenvolvimento dos compartimentos estomacais de bezerros da raça Holandês desaleitados precocemente e recebendo concentrado e volumoso ad libitum a partir do início da segunda e terceira semanas de vida, respectivamente. Foram utilizados 18 animais, distribuídos aleatoriamente em três tratamentos (idade de abate). Foram abatidos seis bezerros ao nascimento (referência), seis aos 50 dias e seis aos 110 dias de vida, para a retirada dos estômagos e mensurações de peso de tecido e volume do órgão e dos compartimentos. Os dados foram submetidos a análises de correlação e regressão, por intermédio do programa estatístico SAS (1996). Foi verificado aumento da capacidade de todos os compartimentos estomacais com o avanço da idade dos bezerros, mas com taxas de crescimento diferenciadas entre os compartimentos. Foram observadas aceleração do crescimento ruminal e retração do crescimento abomasal, com o incremento da idade. Antes dos 50 dias, ocorreu inversão nas proporções dos compartimentos rúmen-retículo e abomaso, enquanto o omaso manteve ritmo constante de desenvolvimento.
\end{abstract}

Palavras-chave: abomaso, desaleitamento precoce, estômago, omaso, rúmen

\section{Stomach Development of Early Weaned Holstein Calf}

\begin{abstract}
The present work was carried out to evaluate the growth of stomach compartments of early weaned Holstein calves. The animals were fed concentrates and hay from the second and third week of age respectively. Eighteen animals were allotted to a completely randomized design, with three treatments (slaughter age). Six calves were slaughtered at birth, at 50 days at 110 days of age; the stomachs were withdrawn and the weight and volume of each compartment measured. Data were submitted to correlation and regression analysis through the SAS statistical program, (1996). An increase of the capacity of all the stomachs compartments was verified with the progress of the age of the calves, but with growth rates differentiated among the compartments. The increase of the reticulorumen growth and the decrease of the abomasum growth with increase of age were observed. Up to 50 days of age the proportion of the reticulo-rumen and abomasum stomach compartments was changed, but the omasum maintained a constant rhythm of development.
\end{abstract}

Key Words: abomasum, early weaned, omasum, rumen, stomach

\section{Introdução}

A criação de bezerros machos de origem leiteira muitas vezes se torna indesejável, devido aos custos elevados na fase de amamentação, desestimulando a exploração da atividade. Entretanto, existem particularidades relativas a técnicas de manejo e características próprias dos animais, inerentes a essa fase de vida, que se consideradas corretamente podem contribuir para alcançar o êxito produtivo.

Os ruminantes no período de aleitamento comportam-se fisiologicamente como animais monogástricos. Forma-se por excitação reflexa do nervo glossofaríngeo, um conduto tubular, chamado de goteira esofagiana, por onde o leite ingerido éconduzido do esôfago direto ao abomaso. Nessa fase a atividade digestiva é exercida pelo abomaso. É a fase mais crítica do ponto de vista nutricional, já que, devido a limitações enzimáticas e à ausência de síntese microbiana, os bezerros apresentam exigências dietéticas mais complexas quanto aos aminoácidos e vitaminas e não utilizam com eficiência certas fontes protéicas e energéticas (Rocha et al., 1999).

Lucci (1989) afirma que o plano nutricional tem influência marcante sobre a velocidade na qual ocorrerá a inversão dos valores de medida entre os compartimentos estomacais rúmen-retículo e abomaso. Quanto maior a quantidade de leite e maior o tempo para fornecê-lo a um bezerro, mais lenta será sua

\footnotetext{
${ }^{1}$ Parte da Dissertação apresentada à Universidade Federal de Santa Maria (UFSM), pelo primeiro autor como um dos requisitos para obtenção do Título de Mestre em Zootecnia.

2 Zootecnista, M.Sc. Doutorando pela Universidade Federal de Lavras (UFLA). Bolsista CNPq. Rua Progresso, 41/801. Lavras, MG. 37200.000. Autor para correspondência. E.mail: pac@ufla.br

${ }^{3}$ Professor do Departamento de Zootecnia da UFSM, RS.

${ }^{4}$ Acadêmico de Zootecnia (UFSM) - Bolsista de iniciação científica CNPq.

${ }^{5}$ Professor da Escola Agrotécnica Federal de São Vicente do Sul, RS.

${ }^{6}$ Médico Veterinário - Colaborador do Setor de Nutrição de Ruminantes da UFSM.
} 
transformação em ruminante funcional.

Em revisão do assunto, Paiva \& Lucci (1972) relatam que os autores são unânimes em afirmar que o desenvolvimento do rúmen em idades precoces está intimamente associado ao consumo de alimentos sólidos. Relatam ainda que os alimentos concentrados através de seu desdobramento em ácidos graxos voláteis no interior do rúmen vão estimular o desenvolvimento da mucosa deste órgão, aumentando o tamanho e o número de papilas ruminais. Quanto ao feno, proporciona maior desenvolvimento do rúmen com respeito à capacidade e aumento do tecido muscular das paredes do órgão, bem como contribui ainda para elevar o $\mathrm{pH}$ no interior do rúmen. Concentrados e fenos fornecidos na mesma dieta, desenvolvem no rúmen uma microflora mais ampla do que se fornecidos isoladamente.

Após o desmame ocorrem modificações na disponibilidade de nutrientes, passando a energia a ser derivada da fermentação ruminal e a proteína a consistir de proteína microbiana e de proteína passante (Funaba et al., 1994).

Orskov (1990) relata ser possível substituir precocemente a proteína do leite por outras proteínas, apesar de que, tecnicamente, essa substituição seja difícil, pois quando a proteína láctea reage com os sucos estomacais forma-se um grande coágulo que posteriormente se desintegra aos poucos. Com outras fontes protéicas esse fenômeno não ocorre. No entanto, a afirmação de Signoretti et al. (1995), de que na fase de aleitamento o leite pode representar até 90 \% do custo de produção, enfatiza a importância na redução de sua quantidade pelos sistemas de desaleitamento precoce. Campos et al. (1991) recomendam utilizar concentrados iniciais corretamente balanceados, a um menor custo e com alta eficiência. Quanto mais cedo se substituir o leite, menor será o custo da criação para o produtor e maior o lucro, pois o leite se destinará para à venda e este animal estará apto a ganhar peso com forragens e com suplementação de concentrados, para ser destinado ao abate.

O objetivo do presente estudo foi avaliar em diferentes idades da fase inicial da vida produtiva, o desenvolvimento dos compartimentos estomacais de bezerros da raça Holandês desaleitados precocemente.

\section{Material e Métodos}

O trabalho foi conduzido no Departamento de Zootecnia da Universidade Federal de Santa Maria, RS. Foram utilizados 18 bezerros da raça Holandês, dos quais seis foram abatidos ao nascimento (no início do experimento). Os 12 animais remanescentes foram mantidos confinados em baias individuais até o abate, ao completarem 50 dias (seis animais) e 110 dias de vida (os seis restantes). O desaleitamento dos bezerros ocorreu gradativamente dos 28 aos 50 dias de vida, sendo que até os 28 dias receberam $4 \mathrm{~L} /$ dia, dos 28 aos 35 dias receberam $3 \mathrm{~L} /$ dia, dos 35 aos 42 dias receberam $2 \mathrm{~L} /$ dia e dos 42 aos 49 dias receberam $1 \mathrm{~L} /$ dia. A partir dos 50 dias de idade não foi administrado leite para os bezerros. No início da segunda semana foi introduzido um concentrado contendo $18 \%$ de PB. Foi ofertado água e feno de alfafa (Medicago sativa) picado ad libitum a partir do início da segunda e terceira semana, respectivamente. No dia determinado para o abate, os animais receberam normalmente a alimentação matinal e após uma hora foram pesados em balança mecânica. Logo a seguir, foram sacrificados, esfolados e realizada a abertura do osso esterno e da cavidade abdominal, para a retirada das vísceras. Imediatamente procedeu-se à ligadura da porção caudal do esôfago com o cárdia, da cranial do duodeno com o esfíncter pilórico, do orifício retículo omasal assim como também do orifício omaso-abomasal.

Por ocasião do abate, os animais foram pesados e amostrados os compartimentos estomacais rúmenretículo, omaso e abomaso, os quais foram pesados como um todo e também separadamente, obtendo-se peso total do órgão bem como dos compartimentos individualmente. Foi determinado o peso do órgão e dos compartimentos vazios, bem como o volume total e por compartimento do estômago com conteúdo, realizado por imersão e deslocamento de água, conforme Tamate et al. (1962).

O delineamento experimental foi inteiramente casualisado, constando de três tratamentos (idades de abate) e seis repetições por tratamento, sendo a unidade experimental representada por um animal.

Os dados foram submetidos a análises de correlação e regressão entre as variáveis, por intermédio do programa SAS (1996).

\section{Resultados e Discussão}

Na Tabela 1, estão as médias dos diferentes pesos, referentes ao momento do abate e nível de significância das características, obtido na análise de variância.

Calculando os ganhos de peso, é verificado que do nascimento aos 50 dias de vida os animais tiveram 
Tabela 1 - Valores médios em kg e nível de significância $(\mathrm{P}>\mathrm{F})$ para peso vivo (PV) e peso de corpo vazio (PCVZ), em bezerros ao nascimento, 50 e 110 dias de vida

Table 1 - Means values $(\mathrm{kg})$ and significance level $(P>F)$ for live weight $(L W)$ and empty body weight (EBW) in calf at birth, 50 and 110 days of age

\begin{tabular}{|c|c|c|c|c|}
\hline \multirow[t]{2}{*}{$\begin{array}{l}\text { Característica } \\
\text { Characteristic }\end{array}$} & \multicolumn{3}{|c|}{$\begin{array}{c}\text { Idade (dias) } \\
\text { Age (days) }\end{array}$} & \multirow[b]{2}{*}{$\mathrm{P}>\mathrm{F}$} \\
\hline & $\begin{array}{c}\text { Nascimento } \\
\text { Birth }\end{array}$ & 50 & 110 & \\
\hline $\begin{array}{l}\mathrm{PV}(\mathrm{kg}) \\
L W\end{array}$ & $40,835^{c}$ & $59,792^{b}$ & $87,025^{a}$ & 0,0001 \\
\hline $\begin{array}{l}\text { PCVZ (kg) } \\
E B W\end{array}$ & $34,130^{c}$ & $48,545^{b}$ & $65,178^{a}$ & 0,0001 \\
\hline $\begin{array}{l}\mathrm{PV} / \mathrm{PCVZ} \\
L W / E B W\end{array}$ & 1,20 & 1,23 & 1,34 & - \\
\hline
\end{tabular}

a, b, c na linha diferem significativamente $(P<0,05)$.

$a, b, c$ in line significant at $P<.05$.

um ganho médio diário (GMD) de 0,379 kg de peso vivo (PV) e 0,288 kg de peso de corpo vazio (PCVZ). Já dos 50 aos 110 dias o GMD foi de 0,454 e 0,277 kg de PV e PCVZ, respectivamente. Observam-se incremento no ganho de peso vivo (GPV) e redução no ganho de peso de corpo vazio (GPCVZ), dentro do intervalo de peso estudado. À medida que aumentou o $\mathrm{PV}$, ocorreu acréscimo na relação PV/PCVZ, o que pode ser explicado pela exclusão do consumo de leite e pelo enchimento do trato digestivo com conteúdo estomacal dos 50 até os 110 dias de vida.

Com o desenvolvimento do animal este comportamento se modifica, como demonstrado por Rocha \& Fontes (1999) que obtiveram uma relação PV/ PCVZ de 1,33 para bezerros Holandês com 150 kg de $\mathrm{PV}$, a partir desse peso, os autores verificaram um decréscimo nessa relação. Da mesma maneira, Ferreira et al. (1999) que trabalharam com bovinos F1 Simental x Nelore, pesando 350; 400; 450 e 500 kg de PV, encontraram relações PV/PCVZ decrescentes na ordem de 1,$22 ; 1,18 ; 1,16$ e 1,14 ; respectivamente. O mesmo resultado foi obtido por Véras et al. (2000), que também encontraram valores decrescentes para a relação PV/PCVZ, quando o PV se elevou de 300 para $450 \mathrm{~kg}$. Os autores obtiveram, com zebuínos, relações de 1,20; 1,17; 1,15 e 1,14 para PV de 300, 350, 400 e $450 \mathrm{~kg}$, respectivamente. O decréscimo na relação PV/PCVZ de animais maduros é decorrente do aumento na proporção de corpo em relação ao conteúdo gastrintestinal, o que pode ser observado a partir dos $150 \mathrm{~kg}$ de PV.
Na Tabela 2, são apresentados os dados correspondentes às mensurações e relações existentes em função do peso do estômago vazio nas três diferentes idades de abate.

Ocorreu comportamento linear crescente $(\mathrm{P}<0,01)$ para os pesos absolutos do estômago (RROA), e seus compartimentos (RR, O e A), em função do aumento de idade dos bezerros até os 110 dias de vida.

Os coeficientes de regressão estimam um aumento estomacal total de $19,78 \mathrm{~g} /$ dia, rumino-reticular de $12,78 \mathrm{~g} / \mathrm{dia}$, omasal de 5,70 g/dia e abomasal de 1,26 g/dia, do nascimento aos 110 dias. Foi observado para o estômago vazio, um acréscimo de 22,7 g/dia do nascimento até os 50 dias reduzindo esse acréscimo para 17,5 g/dia dos 50 aos 110 dias de vida. O rúmenretículo aumentou em 17,1 g/dia até os 50 dias e reduziu o acréscimo de peso do compartimento para 9,4 g/dia dos 50 aos 110 dias de vida. O omaso manteve um aumento crescente de peso com 4,7 e 6,6 g/dia até os 50 e dos 50 aos 110 dias, respectivamente. $\mathrm{O}$ abomaso desenvolveu 0,9 g/dia até os 50 dias e 1,5 g/dia dos 50 aos 110 dias de vida. O baixo crescimento do abomaso até os 50 dias, pode ter sido devido ao fato de os bezerros receberem quantidades decrescentes de leite, já a partir dos 28 dias de vida, determinando forçosamente menor funcionalidade orgânica do ventrículo.

Modesto et al. (1999) desaleitaram precocemente bezerros da raça Holandês e observaram que o rumenretículo e o omaso levaram duas e três semanas, respectivamente, para dobrar de peso. Após a primeira semana a velocidade de crescimento do abomaso caiu, tornando a crescer a partir de 56 dias. O rúmen-retículo aumentou seu peso em oito e o omaso em cinco vezes. Esse comportamento demonstra que a administração de alimentos sólidos, em um sistema de desaleitamento precoce, estimula rápido desenvolvimento muscular do rúmen-retículo até os 50 dias de idade, concordando com Stobo et al. (1966) e Ochoa (1994), que também verificaram esse mesmo comportamento.

Relacionando o peso de tecido estomacal proporcionalmente ao PV, constatou-se um comportamento crescente para o total do estômago e para os compartimentos rúmen-retículo e omaso $(\mathrm{P}<0,01)$ e decrescente para o abomaso $(\mathrm{P}<0,05)$, de acordo com o incremento da idade dos bezerros.

Comportamento semelhante foi observado com relação à porcentagem do PCVZ, com exceção da equação relativa ao abomaso, que não se ajustou ao modelo $(\mathrm{P}>0,05)$. 
Tabela 2 - Valores médios absolutos $(\mathrm{kg})$ e relativos em percentual do peso vivo (\%PV) e percentual do peso de corpo vazio (\%PCVZ), coeficientes de determinação $\left(r^{2}\right)$ e equações de regressão ajustadas para estômago (RROA), rúmen-retículo (RR), omaso (O) e abomaso (A), em função da idade (IDA) em dias

Table 2 - Absolute means values $(\mathrm{kg})$ and relatives in live weight percentage $(\% \mathrm{LW})$ and empty body weight percentage (\%EBW), determination coefficients $\left(r^{2}\right)$ and fitted regression equations for stomach (RROA), reticulo-rumen (RR), omasum (O) and abomasum (A) in according to the days age (IDA)

\begin{tabular}{|c|c|c|c|c|c|}
\hline & \multicolumn{3}{|c|}{$\begin{array}{l}\text { Idade (dias) } \\
\text { Age (days) }\end{array}$} & \multirow[b]{2}{*}{$r^{2}(\%)$} & \multirow[b]{2}{*}{$\begin{array}{l}\text { Equações de regressão } \\
\text { Regression Equation }\end{array}$} \\
\hline & $\begin{array}{l}\text { Nascimento } \\
\text { Birth }\end{array}$ & 50 & 110 & & \\
\hline & & & & $-\mathrm{Kg}$ & \\
\hline RROA & 0,518 & 1,653 & 2,702 & 88,62 & $\ddot{Y}=0,569848+0,019775 * *$ IDA \\
\hline $\mathrm{RR}$ & 0,175 & 1,030 & 1,594 & 83,55 & $\ddot{Y}=0,251154+0,012785 * *$ IDA \\
\hline $\mathrm{O}$ & 0,070 & 0,303 & 0,698 & 88,76 & $\ddot{Y}=0,051449+0,005730 * *$ IDA \\
\hline \multirow[t]{2}{*}{ A } & 0,273 & 0,320 & 0,411 & $\begin{array}{l}50,42 \\
(\% \mathrm{PV})\end{array}$ & $\ddot{Y}=0,267245+0,001260 * *$ IDA \\
\hline & & & & $(\% L W)$ & \\
\hline RROA & 1,28 & 2,76 & 3,08 & 73,92 & $\ddot{Y}=1,519712+0,016017 * *$ IDA \\
\hline $\mathrm{RR}$ & 0,43 & 1,72 & 1,80 & 67,25 & $\ddot{\mathrm{Y}}=0,668381+0,012160 * *$ IDA \\
\hline $\mathrm{O}$ & 0,18 & 0,51 & 0,80 & 85,34 & $\ddot{Y}=0,191621+0,005691 * *$ IDA \\
\hline \multirow[t]{2}{*}{ A } & 0,68 & 0,54 & 0,47 & 33,14 & $\ddot{Y}=0,660361-0,001843 *$ IDA \\
\hline & & & & $\begin{array}{c}(\% \text { PCVZ) } \\
(\% L B W)\end{array}$ & \\
\hline RROA & 1,53 & 3,41 & 4,12 & 78,59 & $\ddot{Y}=1,781522+0,023187 * *$ IDA \\
\hline $\mathrm{RR}$ & 0,51 & 2,12 & 2,42 & 72,66 & $\ddot{\mathrm{Y}}=0,779396+0,016918 * *$ IDA \\
\hline $\mathrm{O}$ & 0,21 & 0,63 & 1,07 & 84,85 & $\ddot{Y}=0,217993+0,007854 * *$ IDA \\
\hline A & 0,81 & 0,66 & 0,63 & - & $\ddot{Y}=0,70$ \\
\hline
\end{tabular}

Trabalhando com bezerros Holandês em um sistema de desaleitamento precoce utilizando uma mistura concentrada + feno de soja perene, Paiva \& Lucci (1972) encontraram, para animais abatidos aos 111 dias de vida, valores de 2,690 kg para RROA, 1,490 kg para RR e 1,200 kg para OA (omaso + abomaso), representando 3,30\% PV para RROA, 1,80\% PV para RR e 1,40\% PV para OA. Os valores apresentados pelos referidos autores foram semelhantes aos obtidos no presente estudo.

Signoretti et al. (1999) encontraram para bezerros da raça Holandês abatidos aos $190 \mathrm{~kg}$ de PV, valores proporcionais ao PCVZ de 2,23; 0,80 e 0,44\% do PCVZ para RR, O e A, respectivamente. No presente estudo os mesmos compartimentos representaram proporções maiores do PCVZ, muito embora os animais apresentassem valores de PV significativamente menores aos 110 dias de vida.

Analisando as proporções dos compartimentos estomacais em relação ao peso de tecido total do estômago (Figura 1), é observado comportamento quadrático positivo $(\mathrm{P}<0,01)$, em função da idade, para rúmen-retículo, com ponto de máximo estimado de 65,5\% do estômago aos 74 dias e comportamento quadrático negativo $(\mathrm{P}<0,01)$ para o abomaso, com ponto de mínimo estimado de 12,48 \% do estômago aos 86 dias de vida. A proporção do omaso em relação ao total do estômago apresentou um comportamento linear crescente, em função da idade, o que concorda com Paiva \& Lucci (1972), Coelho da Silva \& Leão (1979), Churc (1993) e Coelho et al. (1999) e indica inversão no peso proporcional dos compartimentos estomacais $\mathrm{RR}$ e A, antes dos 50 dias de vida em bezerros desaleitados precocemente.

Ao nascimento o abomaso ocupa maior proporção, mas a partir do momento em que os bezerros começam a ingerir alimento sólido, o rúmen-retículo passa a ocupar maior peso proporcional de tecidos. Para verificar as modificações estomacais, em função do PV, foram ajustadas as equações constantes na Tabela 3.

Aplicando-se a equação ajustada para RROA, estimou-se o peso absoluto de tecido estomacal para os bezerros abatidos no presente experimento. Os valores estimados de 0,$52 ; 1,65$ e $2,70 \mathrm{~kg}$ ao nascimento, 50 e 110 dias, foram bastante próximos aos valores observados (Tabela 3). Tal fato demonstra que as mesmas podem ser utilizadas com segurança, 


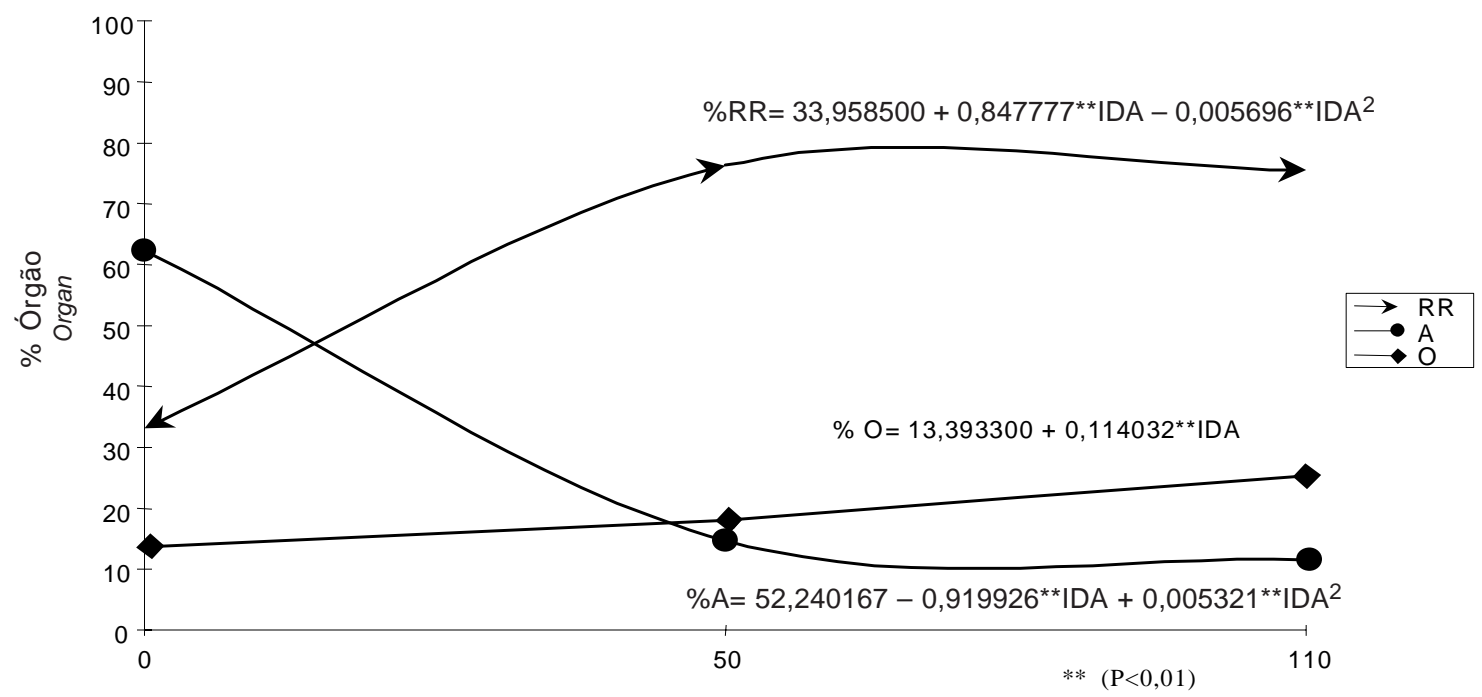

Dias de vida

Days of age

Figura 1 - Desenvolvimento proporcional dos compartimentos estomacais, em relação ao peso de tecido total do estomago de bezerros ao nascimento, 50 e 110 dias de vida.

Figure 1 - Proportional development $(\mathrm{kg})$ of stomachs compartments of calf at birth, 50 and 110 days of age.

para animais da mesma raça, sexo, peso e sob mesmas condições.

Na Tabela 4, encontram-se os valores correspondentes às mensurações e relações existentes, em função do volume absoluto do estômago (com conteúdo) dos bezerros nas três diferentes idades de abate.

O volume total do estômago (RROA) e de seus compartimentos (RR, O e A) e a relação percentual existente entre estes e o PV e PCVZ aumentaram linearmente $(\mathrm{P}<0,01)$ com o incremento de idade dos animais. Através das equações ajustadas, estima-se um aumento da capacidade volumétrica na ordem de $238 \mathrm{~mL} /$ dia para RROA, de $203 \mathrm{~mL} /$ dia para RR, de $16 \mathrm{~mL} /$ dia para $\mathrm{O}$ e de $20 \mathrm{~mL} /$ dia para A.

O acentuado aumento de volume total do estômago com conteúdo, observado do nascimento aos 110 dias de vida demonstra grande desenvolvimento da capacidade estomacal, quando os bezerros passam a receber uma dieta sólida no sistema de desaleitamento
Tabela 3 - Equação de regressão ajustada, coeficiente de determinação $\left(r^{2}\right)$ e nível de significância $(P>F)$ para estimar o peso absoluto de estômago total (RROA) e dos compartimentos rúmen-retículo (RR), omaso (O) e abomaso (A), em função do peso vivo (PV) em bezerros da raça Holandês do nascimento aos 110 dias de vida

Table 3 - Fitted regression equation, correlation coefficients $(r)$, determination coefficients $\left(r^{2}\right)$ and significance level $(P>F)$ for estimating the absolute weight $(\mathrm{kg})$ of total stomach (RROA) and reticulo-rumen (RR), omasum (O) and abomasum (A) compartments in according to live weight $(L W)$ in Holstein calf at birth, 50 and 110 days of age

\begin{tabular}{lcc}
\hline $\begin{array}{l}\text { Equação ajustada } \\
\text { Fitted equation }\end{array}$ & $\mathrm{r}^{2}(\%)$ & $\mathrm{P}>\mathrm{F}$ \\
\hline $\mathrm{RROA}(\mathrm{kg})=-1,1978+0,0451 * \mathrm{PV}(\mathrm{kg})$ & 93,66 & 0,0001 \\
$* L W(K G)$ & & \\
$\mathrm{RR}(\mathrm{kg})=-0,9138+0,0295 * \mathrm{PV}(\mathrm{kg})$ & 90,46 & 0,0001 \\
$* L W(K G)$ & & \\
$\begin{array}{l}\mathrm{O}(\mathrm{kg})=-0,4262+0,0125 * \mathrm{PV}(\mathrm{kg}) \\
* L W(K G)\end{array}$ & 86,04 & 0,0001 \\
$\mathrm{~A}(\mathrm{~kg})=0,1421+0,0031 * \mathrm{PV}(\mathrm{kg})$ & 60,94 & 0,0001 \\
$* L W(K G)$ & & \\
\hline
\end{tabular}


Tabela 4 - Valores médios absolutos (litro) e relativos em percentual do peso vivo (\% PV) e percentual do peso de corpo vazio (\% PCVZ), coeficientes de determinação $\left(r^{2}\right)$ e equações de regressão ajustadas para estômago (RROA), rúmen-retículo (RR), omaso (O) e abomaso, em função da idade (IDA) em dias

Table 4 - Absolute means values (liter) and relatives in live weight percentage (\% LW) and empty body weight percentage (\% EBW), determination coefficients $\left(r^{2}\right)$ and fitted regression equations for stomach (RROA), reticulo-rumen (RR), omasum (O) and abomasum (A) in according to the days age (IDA)

\begin{tabular}{|c|c|c|c|c|c|}
\hline & \multicolumn{3}{|c|}{$\begin{array}{l}\text { Idade (dias) } \\
\text { Age (days) }\end{array}$} & \multirow[b]{2}{*}{$r^{2}(\%)$} & \multirow[b]{2}{*}{$\begin{array}{c}\text { Equações de regressão } \\
\text { Regression Equation }\end{array}$} \\
\hline & $\begin{array}{c}\text { Nascimento } \\
\text { Birth }\end{array}$ & 50 & 110 & & \\
\hline & & & & Litro & \\
\hline & & & & Liter & \\
\hline RROA & 1,66 & 11,93 & 27,84 & 93,61 & $\ddot{Y}=1,072234+0,238896 * *$ IDA \\
\hline $\mathrm{RR}$ & 0,64 & 9,83 & 22,88 & 90,58 & $\ddot{Y}=0,308700+0,202701 * *$ IDA \\
\hline $\mathrm{O}$ & 0,18 & 0,75 & 1,95 & 80,74 & $\ddot{Y}=0,104652+0,016142 * *$ IDA \\
\hline \multirow[t]{3}{*}{ A } & 0,85 & 1,34 & 3,01 & 73,12 & $\ddot{Y}=0,673590+0,019891 * *$ IDA \\
\hline & & & & $(\% \mathrm{PV})$ & \\
\hline & & & & $(\% L W)$ & \\
\hline RROA & 4,18 & 20,13 & 32,00 & 90,26 & $\ddot{Y}=5,379079+0,251118 * *$ IDA \\
\hline $\mathrm{RR}$ & 1,62 & 16,59 & 26,16 & 86,53 & $\ddot{Y}=3,007967+0,220907 * *$ IDA \\
\hline $\mathrm{O}$ & 0,47 & 1,29 & 2,30 & 66,58 & $\ddot{Y}=0,462493+0,016671 * *$ IDA \\
\hline \multirow[t]{3}{*}{ A } & 2,13 & 2,25 & 3,55 & 34,85 & $\ddot{Y}=1,943348+0,013158 * *$ IDA \\
\hline & & & & (\% do PCVZ) & \\
\hline & & & & $(\% E B W)$ & \\
\hline RROA & 4,99 & 24,81 & 42,69 & 91,49 & $\ddot{Y}=5,961775+0,341259 * *$ IDA \\
\hline $\mathrm{RR}$ & 1,94 & 20,44 & 34,91 & 88,38 & $\ddot{Y}=3,211416+0,297787 * *$ IDA \\
\hline $\mathrm{O}$ & 0,56 & 1,60 & 3,06 & 69,00 & $\ddot{Y}=0,520925+0,022809 * *$ IDA \\
\hline A & 2,55 & 2,78 & 4,72 & 42,86 & $\ddot{Y}=2,271549+0,020202 * *$ IDA \\
\hline
\end{tabular}

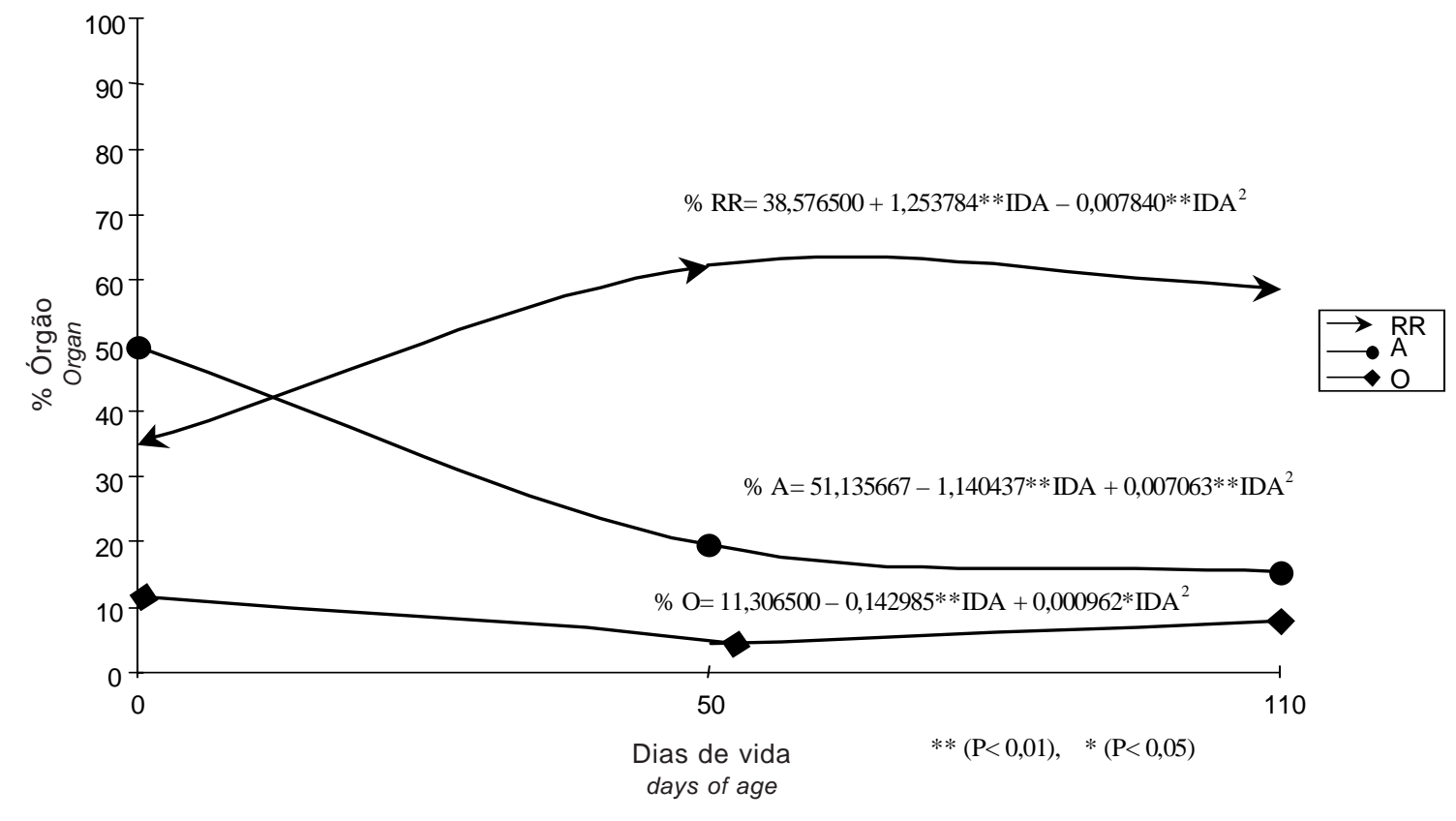

Figura 2 - Desenvolvimento volumétrico proporcional dos compartimentos estomacais dos bezerros ao nascimento, 50 e 110 dias de vida.

Figure 2 - Volumetric proportional development of stomachs compartments of calf at birth, 50 and 110 days of age. 
precoce, concordando com Harrison \& Warner (1961); Tamate et al. (1962) e Paiva \& Lucci (1972), os quais observaram grande aumento da capacidade ruminal antes de quatro semanas de idade, em bezerros que receberam uma dieta à base de alimentos sólidos. No presente estudo o rúmen-retículo aumentou a sua capacidade volumétrica em 15 vezes até os 50 dias e 36 vezes até os110 dias de vida.

A proporção volumétrica entre os compartimentos estomacais e o estômago como um todo (Figura 2), apresentou comportamento quadrático positivo ( $\mathrm{P}<0,01)$, em função da idade, para RR com um ponto de máximo estimado de $88,70 \%$ do volume estomacal aos 80 dias e comportamento quadrático negativo $(\mathrm{P}<0,01)$ para o abomaso, com um ponto de mínimo estimado de 5,10\% do volume total aos 81 dias de vida. A proporção do omaso em relação ao volume total do estômago apresentou comportamento quadrático negativo $(\mathrm{P}<0,05)$, com ponto de mínimo estimado de 5,99\% do volume aos 74 dias.

Foi observada inversão dos valores de capacidade volumétrica proporcional dos diferentes compartimentos do estômago dos bezerros desaleitados precocemente, que passaram a ingerir alimento sólido desde as primeiras semanas de vida. Tal resultado concorda com Church (1971) e Lucci (1989), que relatam valores proporcionais de rúmen-retículo com cerca de $30 \%$ e omaso mais abomaso de $70 \%$ do volume total dos compartimentos, ao nascimento. Os mesmos autores afirmam que um animal adulto apresenta esses valores invertidos, sendo que o rúmenretículo perfazem até mais de $80 \%$, restando para omaso e abomaso menos de $20 \%$ do volume total. Igualmente, Coelho da Silva \& Leão (1979) relatam valores de 30\% para rúmen-retículo e $70 \%$ para

Tabela 5 - Equação de regressão ajustada, coeficiente de determinação $\left(r^{2}\right)$ e nível de significância $(P>F)$ para estimar o volume absoluto em litros (L) de estômago total (RROA) e dos compartimentos rúmen-retículo (RR), omaso (O) e abomaso (A), em função do peso vivo (PV) em bezerros Holandês do nascimento aos 110 dias de vida

Table 5 - Fitted regression equation, determination coefficients $\left(r^{2}\right)$ and significance level $(P>F)$ for estimating the absolute volume $(L)$ of total stomach (RROA) and reticulo-rumen (RR), omasum (O) and abomasum (A) compartments in according to live weight (LW) in Holstein calf at birth, 50 and 110 days of age

\begin{tabular}{lcc}
\hline $\begin{array}{l}\text { Equação ajustada } \\
\text { Fitted equations }\end{array}$ & $\mathrm{r}^{2}(\%)$ & $\mathrm{P}>\mathrm{F}$ \\
\hline $\begin{array}{l}\text { RROA }(\mathrm{L})=-18,5033+0,5166 * \mathrm{PV}(\mathrm{kg}) \\
{ }^{*} L W(\mathrm{~kg})\end{array}$ & 88,88 & 0,0001 \\
$\begin{array}{l}\mathrm{RR}(\mathrm{L})=-16,6852+0,4445 * \mathrm{PV}(\mathrm{kg}) \\
{ }^{*} L W(\mathrm{~kg})\end{array}$ & 88,43 & 0,0001 \\
$\begin{array}{l}\mathrm{O}(\mathrm{L})=-1,0342+0,0320 * \mathrm{PV}(\mathrm{kg}) \\
*\end{array}$ & 64,30 & 0,0001 \\
$\begin{array}{l}\text { A }(\mathrm{L})=-0,759) \\
* L W(\mathrm{~kg})\end{array}$ & 59,63 & 0,0002 \\
\hline
\end{tabular}

Tabela 6 - Coeficientes de correlação ( $r$ ) e níveis de significância (P>F) entre o peso absoluto (kg) e o volume (L) de estômago total (RROA), e dos compartimentos rúmen-retículo (RR), omaso (O) e abomaso (A)

Table 6 - Correlation coefficients $(r)$ and significance level $(P>F)$ among absolute weight $(\mathrm{kg})$ and volume $(L)$ of total stomach $(R R O A)$ and reticulo-rumen $(R R)$, omasum $(O)$ and abomasum $(A)$ compartments

\begin{tabular}{|c|c|c|c|c|c|c|c|c|}
\hline r & $\begin{array}{c}\text { RROA } \\
\text { (kg) }\end{array}$ & $\begin{array}{c}\mathrm{RR} \\
\text { (kg) }\end{array}$ & $\begin{array}{c}\mathrm{O} \\
(\mathrm{kg})\end{array}$ & $\begin{array}{c}\mathrm{A} \\
(\mathrm{kg})\end{array}$ & $\begin{array}{l}\text { RROA } \\
\text { (L) }\end{array}$ & $\begin{array}{l}\mathrm{RR} \\
(\mathrm{L})\end{array}$ & $\begin{array}{c}\mathrm{O} \\
(\mathrm{L})\end{array}$ & $\begin{array}{c}\text { A } \\
\text { (L) }\end{array}$ \\
\hline RROA & $* * 1,0000$ & 0,9905 & 0,9415 & 0,8040 & 0,9648 & 0,9648 & 0,8208 & 0,7806 \\
\hline (kg) & $* 0,0000$ & 0,0001 & 0,0001 & 0,0001 & 0,0001 & 0,0001 & 0,0001 & 0,002 \\
\hline $\mathrm{RR}$ & - & 1,0000 & 0,8926 & 0,7838 & 0,9430 & 0,9534 & 0,7501 & 0,6941 \\
\hline (kg) & & 0,0000 & 0,0001 & 0,0001 & 0,0001 & 0,0001 & 0,003 & 0,0014 \\
\hline $\mathrm{O}$ & - & - & 1,0000 & 0,6824 & 0,9353 & 0,9057 & 0,9425 & 0,9011 \\
\hline (kg) & & & 0,0000 & 0,0018 & 0,0001 & 0,0001 & 0,0001 & 0,0002 \\
\hline A & - & - & - & 1,0000 & 0,7824 & 0,8024 & 0,5732 & 0,5121 \\
\hline (kg) & & & & 0,0000 & 0,0001 & 0,0001 & 0,0129 & 0,0298 \\
\hline RROA & - & - & - & sits & 1,0000 & 0,9950 & 0,8705 & 0,8255 \\
\hline (L) & - & & & & 0,0000 & 0,0001 & 0,0001 & 0,0001 \\
\hline $\mathrm{RR}$ & - & - & - & - & - & 1,0000 & 0,8195 & 0,7664 \\
\hline (L) & & & & & & 0,0000 & 0,0001 & 0,0002 \\
\hline $\begin{array}{l}\mathrm{O} \\
\text { (L) }\end{array}$ & - & - & - & - & - & - & $\begin{array}{l}1,0000 \\
0,0000\end{array}$ & $\begin{array}{l}0,9596 \\
0,0001\end{array}$ \\
\hline $\begin{array}{l}\text { A } \\
\text { (L) }\end{array}$ & - & - & - & - & - & - & - & $\begin{array}{l}1,0000 \\
0,0000\end{array}$ \\
\hline
\end{tabular}


omaso mais abomaso ao nascimento e 84\% para rúmenretículo e $16 \%$ para omaso mais abomaso aos quatro meses de idade.

Na Tabela 5, são apresentadas as equações de regressão ajustadas para predizer o volume do estômago, a partir do peso vivo do animal.

Quando foram correlacionadas as variáveis medidas em peso (kg) com as variáveis medidas em volume (L) encontraram-se elevados e significativos coeficientes de correlação (Tabela 6) para o estômago como um todo (RROA) assim como para os compartimentos rúmen-retículo (RR), omaso (O) e abomaso (A).

Com exceção dos valores obtidos para o abomaso, as demais correlações e níveis de significância $(P>F)$ indicam existir alta relação entre o peso e o volume do estômago total e dos compartimentos estomacais, até os 110 dias de idade.

\section{Conclusões}

É verificado aumento da capacidade de todos os compartimentos estomacais com o avanço da idade dos bezerros, no entanto ele ocorre de maneira distinta, com taxas de crescimento diferenciadas entre os compartimentos.

Com o incremento da idade dos bezerros ocorre uma aceleração do crescimento ruminal e uma retração do crescimento abomasal.

A inversão nas proporções dos compartimentos estomacais ocorre antes dos 50 dias em bezerros desaleitados precocemente.

\section{Literatura Citada}

CAMPOS, O.F.; MATOS, L.L.; RODRIGUES, A.A. Bezerros: quando definir o desaleitamento. Balde Branco, v.27, n.314, p.24-26, 1991.

CHURCH, D.C. Fisiologia digestiva y nutrición de los ruminantes. Zaragoza: Acríbia, 1971. 544p.

CHURCH, D.C. El rumiante: fisiología digestiva y nutrición. Zaragoza: Acríbia, 1993. 641p.

COELHO DA SILVA, J.F.; LEÃO, M.I. Fundamentos de nutrição de ruminantes. São Paulo: Livroceres, 1979. 380p.

COELHO, S.G.; SATURNINO, H.M.; GUEDES, R.M. et al. Desaleitamento de bezerros aos 30 dias de idade e alimentação com ou sem volumoso até 60 dias: II- Desenvolvimento do estômago até os 90 dias de idade. In: REUNIÃO ANUAL DA SOCIEDADE BRASILEIRA DE ZOOTECNIA, 36., 1999, Porto Alegre. Anais ... Porto Alegre, 1999. p.237.

FERREIRA, M.A.; VALADARES FILHO, S.C.; COELHO DA SILVA, J.F. et al. Composição corporal e exigências líquidas de proteína e energia para ganho de peso de bovinos F1 Simental x Nelore. Revista Brasileira de Zootecnia, v.28, n.2, p.352-360, 1999.

FUNABA, M.; KAGIYAMA, K.; IRIKI, T. et al. Changes nitrogen balance with age in calves weaned at 5 or 6 weeks of age. Journal of Animal Science, v.72, n.3, p.732-738, 1994.

HARRISON, H; WARNER, G. A quantitative in vivo study of rumen capacity as influenced by milk and dry feed consumption. Journal of Dairy Science, v.44, p.1178-1190, 1961.

LUCCI, C.S. Bovinos leiteiros jovens. São Paulo: Nobel, 1989. 371p.

MODESTO, E.C.; MANCIO, A.B.; MENIM, E. et al. Utilização de colostro fermentado para bezerros: Desenvolvimento alométrico. In: REUNIÃO ANUAL DA SOCIEDADE BRASILEIRA DE ZOOTECNIA, 36., 1999, Porto Alegre. Anais ... Porto Alegre: Sociedade Brasileira de Zootecnia, 1999. p. 230

OCHOA, S.M.; NEIVA, R.S.; GIRÃO, L.C.F. et al. Desenvolvimento ruminal e papilar em bezerros mestiços (Holandês-Zebu). Revista Ciência e Prática, v.18, n.3, p.320-325, 1994.

ORSKOV, E.R. Alimentación de los ruminantes. Acríbia, Zaragoza, 1990. p.10.

PAIVA, J.A.J.; LUCCI, C.S. Alimentação de bezerros com mistura concentrada comum + feno de soja perene. II Desenvolvimento dos pró-ventrículos. Boletim da Industria Animal, v.29, n.1, p.151-159, 1972.

ROCHA, E.O.; FONTES, C.A.A.; PAUlinO, M.F. et al. Influência da idade de desmama e de início do fornecimento do volumoso a bezerros sobre a digestibilidade de nutrientes e o balanço de nitrogênio, pós-desmama. Revista Brasileira de Zootecnia, v.28, n.1, p.143-147, 1999.

ROCHA, E.O.; FONTES, C.A.A. Composição corporal, composição do ganho de peso e exigências nutricionais de novilhos de origem leiteira. Revista Brasileira de Zootecnia, v.28, n.1, p.159-168, 1999.

STATISTICAL ANALYSIS SYSTEM - SAS. User's guide: Stactistcs. Version 6.11. Cary: 1996.

SIGNORETTI, R.D.; CASTRO, A.C.G.; COELHO DA SILVA, J.F. et al. Utilização do farelo de gérmen de milho no concentrado inicial de bezerros de raças leiteiras em sistemas de desaleitamento precoce. Revista Brasileira de Zootecnia, v.24, n.5, p.841-851, 1995.

SIGNORETTI, R.D.; ARAÚJO, G.G.L.; COELHO DA SILVA, J.F. et al. Composição física da carcaça de bezerros da raça holandesa alimentados com dietas contendo diferentes níveis de concentrado. Revista Brasileira de Zootecnia, v.28, n.4, p.883-888, 1999.

STOBO, I.J.F; ROY, J.H.B; GASTON, H.J. Rumen development in the calf. 1-The effect of diets containing different proportions of concentrates to hay on rumen development. British Journal of Nutrition, v.20, n.2, p.171-191, 1966.

TAMATE, H.; McGILLIARD, A.D.; JACOBSON, N.L. et al. Effect of various dietaries on the anatomical development of the stomach in the calf. Journal of Dairy Science, v.45, p.408-420, 1962.

VÉRAS, A.S.C.; VALADARES FILHO, S.C.; COELHO DA SILVA, J.F. et al. Predição da composição corporal de bovinos não-castrados, alimentados com rações contendo diferentes níveis de concentrado. In: REUNIÃO ANUAL DA SOCIEDADE BRASILEIRA DE ZOOTECNIA, 37., 2000, Viçosa, MG. Anais... Viçosa: Sociedade Brasileira de Zootecnia, 2000. p.473. 